\title{
[Industrial paper]
}

\section{Combustion Engine Mechanism Analyses Using SimulationX}

\author{
Tomohide Hirono $^{1}$ Takanori Watanabe ${ }^{2}$ \\ ${ }^{1,2}$ CAE Research \& Development Center, NewtonWorks Corp., Japan, \\ \{hirono.tomohide, twatanabe @newtonworks.co.jp
}

\begin{abstract}
When we apply more efficient combustion profile using advanced mechanisms to ICEs, vehicles with ICEs can exceed BEVs from the view of total environmental performance. This paper illustrates SimulationX, a Modelica simulation tool, can be a tool for the developments of advanced mechanical systems such as new valve trains and cranking systems. This paper also shows a case of FMI co-simulation of a cam phaser between the tool and another hydraulic simulation tool which is used to model a conventional hydraulic system of an existing ICE.
\end{abstract}

Keywords: Internal Combustion Engine, Valve Train, Cam Phaser, Variable Compression Ratio, FMI, CoSimulation

\section{Introduction}

The total (i.e. from well to wheel) environmental performance of vehicles with ICEs (Internal Combustion Engines), can exceed BEVs (Battery Electric Vehicles) when the efficiency of combustion is improved and advanced mechanical systems are required to realize the combustion profiles. ${ }^{1}$ Resources to develop ICEs are, however, not sufficient since automotive OEMs are also required to develop xEVs in parallel. To improve the productivity of developments of the systems, more efficient tools to confirm ideas of engineers are in demand. A cam phase change mechanism (cam phaser), variable valve lift mechanisms and a variable compression ratio system are modeled using SimulationX, a Modelica simulation tool, and illustrated in this paper to show the availability of the tool to realize faster development at earlier stages in MBD cycles.

This paper also explains availabilities of FMI between two hydraulic simulation tools. Most of the conventional hydraulic tools used by automotive OEMs to model hydraulic systems of ICEs are single domain tools. The Modelica tool is a multi-domain simulation tool and it can realize simulation of hydraulic-mechanical interactions. The existing hydraulic circuit modeled by a conventional tool can be

\footnotetext{
${ }^{1}$ Mr. Hitomi presented at Automotive World 2018 in Tokyo.
}

slightly modified to connect to the Modelica tool to supply hydraulic pressure to drive a hydraulic CAM phaser modeled in the tool. The scheme is realized using FMI technology and illustrated here.

\section{Valve Trains}

Changing combustion timing and adding air mass flow flown into cylinders are ways to increase efficiency of ICEs. They are realized by changing valve lifts in timing and in traveling distances. Cam phasers are used to change lifts in timing. A single valve with multiple cam mechanism or adding intermediate arm between a cam and a valve mechanism is used both in timing and lift changes.

\subsection{Cam Phaser}

A hydraulic cam phaser is shown in Figure 1 and the diagram of total cam phaser system modeled with the tool is shown in Figure 2.

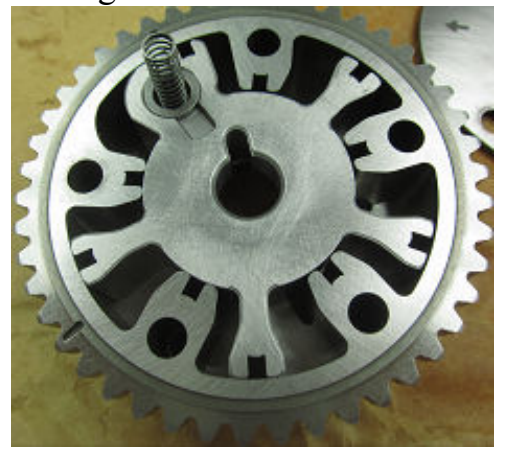

Figure 1 Hydraulic Cam Phaser (5 x 2 Rooms)

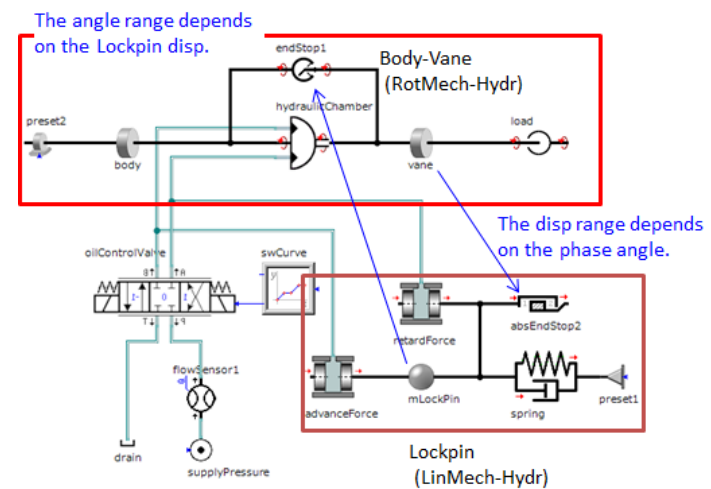

Figure 2 Diagram of Cam Phaser System 
This type of hydraulic cam phasers has a body with a sprocket and inner vanes and a rotor with outer vanes. The sprocket is driven by a chain to/from a crank shaft sprocket and the rotor is connected to a cam shaft. There are some fluid volumes (rooms) between the body vanes and the rotor vanes into which lubricant flows to realize to advance or retard phases. A valve controls flow direction to fluid volumes into which the pressurized fluid flows. A lockpin is used to fix the phaser angle when the pressure is lower than a certain level to avoid free motion in low pressurized states such as starting up the engine. The phaser rotor rotates by the fluid pressure relative to the crank shaft rotations then phase advancement or retardation occurs. Figure 3 shows the results of the model. At $\mathrm{t}=0$, there is no pressure in the circuits and it gradually increases to $\mathrm{t}=0.1[\mathrm{~s}]$. At $\mathrm{t}=0.2[\mathrm{~s}]$ advance signal changes to 1 then the hydraulic pressure in advance rooms goes up to $100[\mathrm{kPa}]$ to rotate the vane. The displacement of the lockpin is $5[\mathrm{~mm}]$ and it indicates relative motion between the rotor and the body. The relative rotation stops at about $\mathrm{t}=0.95[\mathrm{~s}]$ since the rotor contacts to the other side of the body mechanically. The retardant volume is almost zero now. The retardant motion occurs at $\mathrm{t}=1.2[\mathrm{~s}$ ] triggered by a ' -1 ' advance signal and a motion to the opposite direction is observed. The lockpin moves back to $1[\mathrm{~mm}]$ position at $\mathrm{t}=2.0$ [s]. The lockpin connects the rotor and the body mechanically.
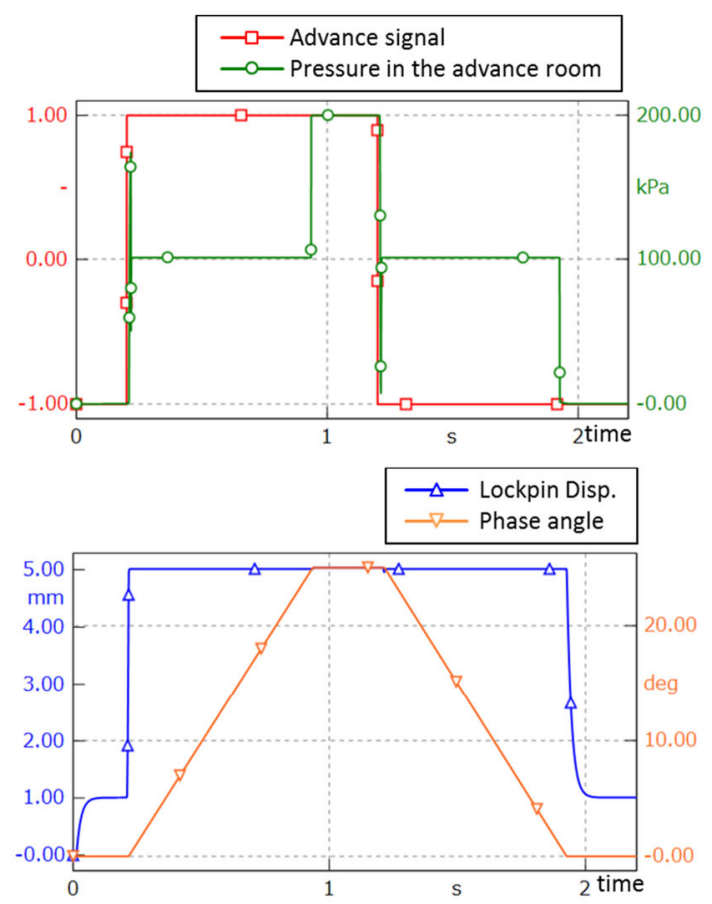

Figure 3 Simulation Results. (Top) Input Signal to Advance and Pressure in the Advance Rooms (Bottom) Lockpin Displacement and Phase Angle

\subsection{Valve Mechanism}

\subsubsection{Multiple Cam Change Over Mechanism MCCOM}

This type of mechanism has two cams with different profiles for a valve. One of the cams is selected to push the valve according to the engine rotational speed and/or load required (Figure 4).

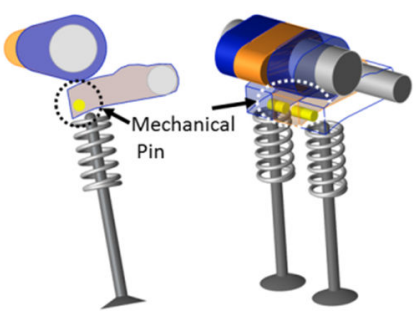

Figure 4 MCCOM (Blue: Low Lift Cam Orange: High Lift Cam)

This mechanism is modeled as seen in Figure 5 A pin is used to fix the selected cam to work. The traveling time of the pin has direct relation to the response of the cam selection. A cam element developed for the tool is used here.

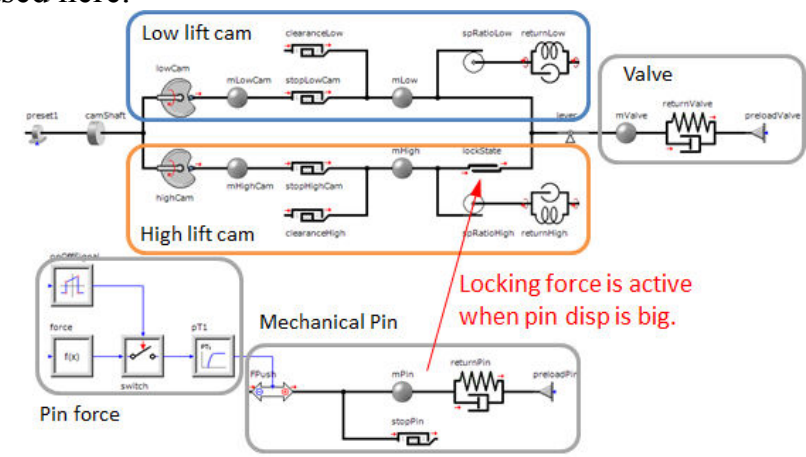

Figure 5 Diagram of MCCOM

The Figure 6 shows the valve stroke. The first stroke is invoked by the low lift cam and the second stroke is invoked by the high lift cam.

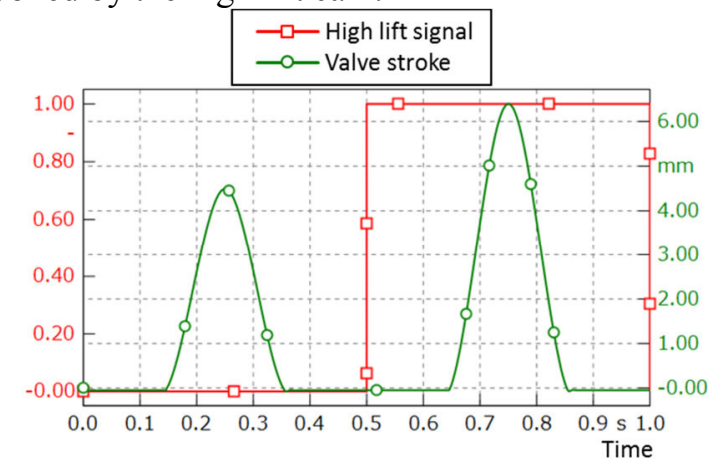

Figure 6 Valve Strokes 


\subsubsection{Intermediate Arm and Cam}

This type of the system has a set of an intermediate arm and a cam between a cam attached to a cam shaft and a rocker arm. The traveling distance (stroke) of the valve can be changed continuously by setting the angle of the intermediate arm which I controlled by a control shaft driven by an electric motor. The mechanism can be modeled using MBS (Multi Body System) Library and contact elements of the tool as seen in Figure 7.
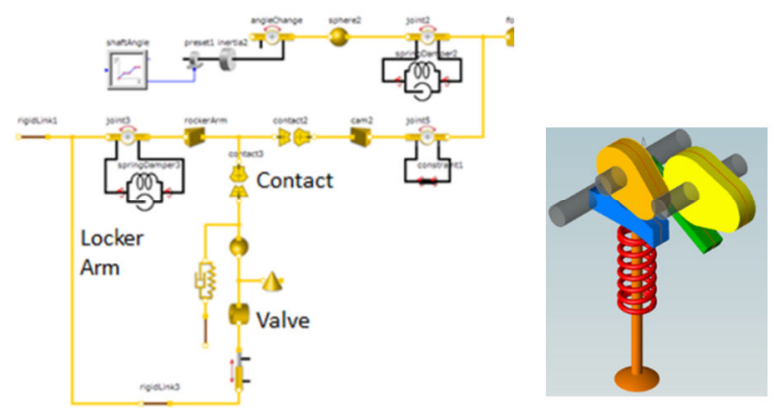

Figure 7 (Left) Diagram of Valve Train with Intermediate Cam and Arm.

(Right) Mechanism of Intermediate Arm and Cam.

The stroke changes are seen in Figure 8 at $\mathrm{t}=2.5$ and $4.5[\mathrm{~s}]$ when control shaft angle is changed.

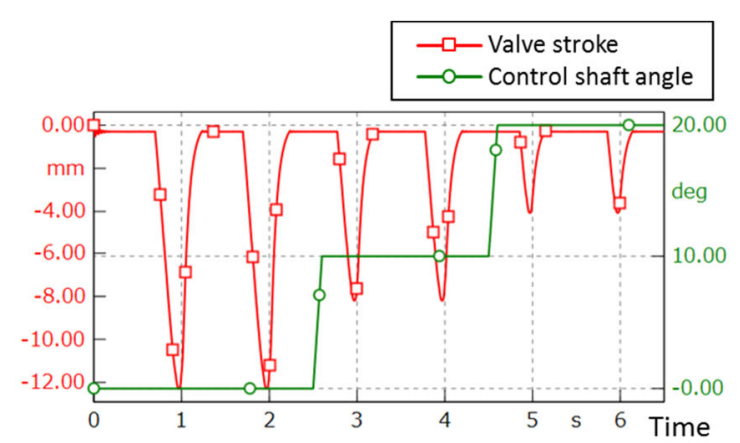

Figure 8 Changes of Valve Stroke by Control Shaft Angle

\section{Variable Compression Ratio System}

One of different approaches to make ICEs more efficient is changing compression ratios. Conventional ICEs have crank systems and the traveling distance (stroke) of pistons are fixed. This type of mechanisms can change the distance by an additional multiple-link (Figure 9). The diagram modeled by the tool is seen in Figure 10. Mechanical engineers can model this kind of systems easily using mechanical 3D-CAD after each of the components of the links is designed as a CAD model but they have difficulties to calculate required torque to rotate the linkage at earlier stage of design cycles. The tool can be used to estimate the torque in many circumstances of engines in practical uses. The Figure 11 shows how its compression ratio changes by its control shaft angle. Optimal quantities of fuel will be supplied to the cylinders in order to obtain required power to drive the vehicle which are dependent on then current compression ratio. Required power to rotate the control shaft can be also obtained when pressure in the cylinder is obtained.

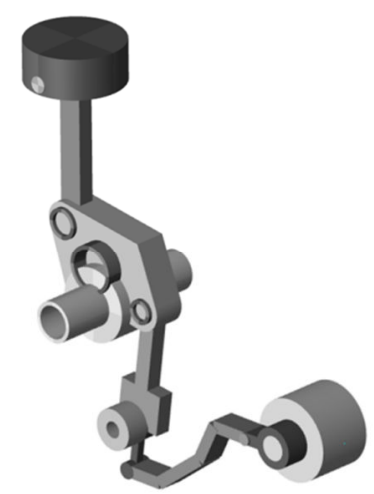

Figure 9 Variable Compression Ratio Mechanism

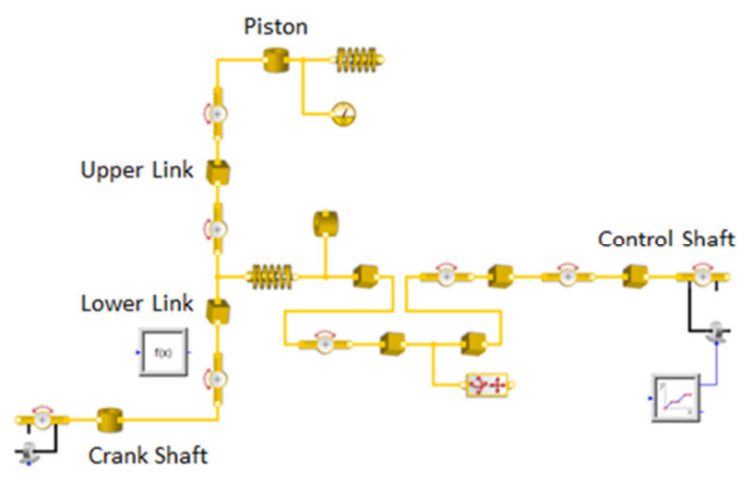

Figure 10 Diagram of VCR Using MBS

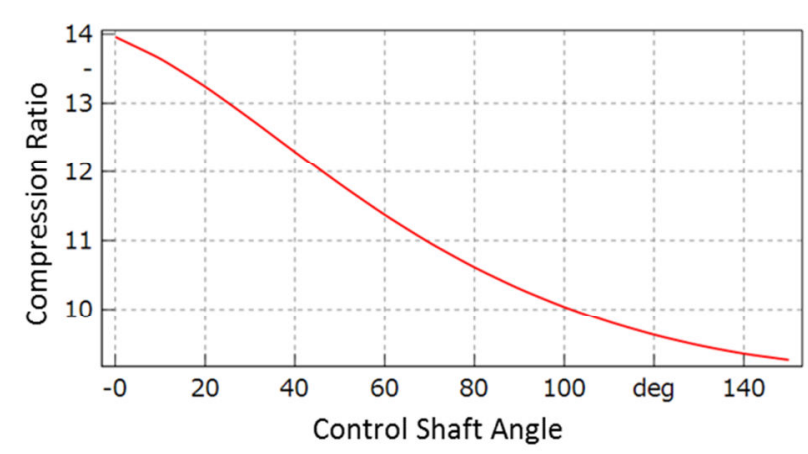

Figure 11 Compression Ratio Change by Shaft Angle

\section{Co-Simulation of Hydraulic Tools with FMI}

ICEs have hydraulic circuits to lubricate between pistons and cylinders, crank shaft bearings and the shafts, cam mechanisms. Lubricant is also used for sealing and cooling purposes. The circuits used to be 
designed and analyzed using conventional flow simulation tools. Those tools are, in most cases, designed mainly for flow and/or thermal analysis purposes and have not enough capability to simulate mechanical motion. The OEMs, however, have a large amount of legacy data generated with the fluidic/thermal tools. The engineers of the OEMs have interests in using the models to survey additional functions such as cam phasers, variable compression mechanisms and so on whose motions are driven by the power supplied by hydraulic systems. We would like to discuss a model - i) the existing circuit model supplies fluid to drive a new mechanical component, ii) the new mechanism is modeled by a different tool and iii) a co-simulation with the two tools is performed using FMI technology here in this section. Two different tools are employed for the co-simulation, one is SimulationX and the other is Flowmaster. Please note the diagrams of the models shown here in this paper are expressed with a single tool (SimulationX) due to intellectual property reasons. The model used for the co-simulation is different from the one seen in Figure 12, actually.

\subsection{Connections for CoSimulation}

We added a junction at a point marked as $\mathrm{A}$ in the Figure 12 to supply oil to a cam phaser, the new mechanism. The oil supplied to the cam phaser goes down to an oil pan via volumes for advancement or retardation. The whole system diagram that we model is seen in Figure 13. The results are shown in Figure 14. It shows the pressure at the main gallery (MG) changes by the phase switch because of additional flow to the cam phaser.

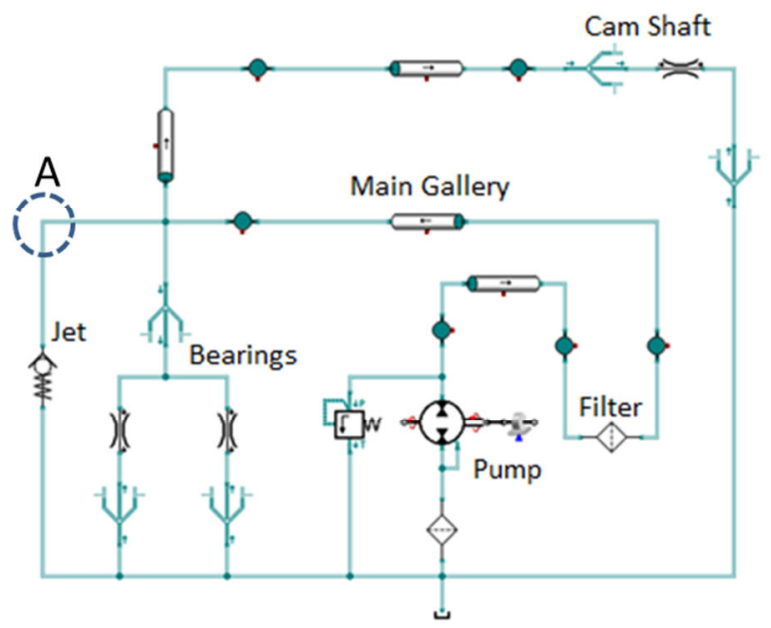

Figure 12 Diagram of Existing Hydraulic Circuit

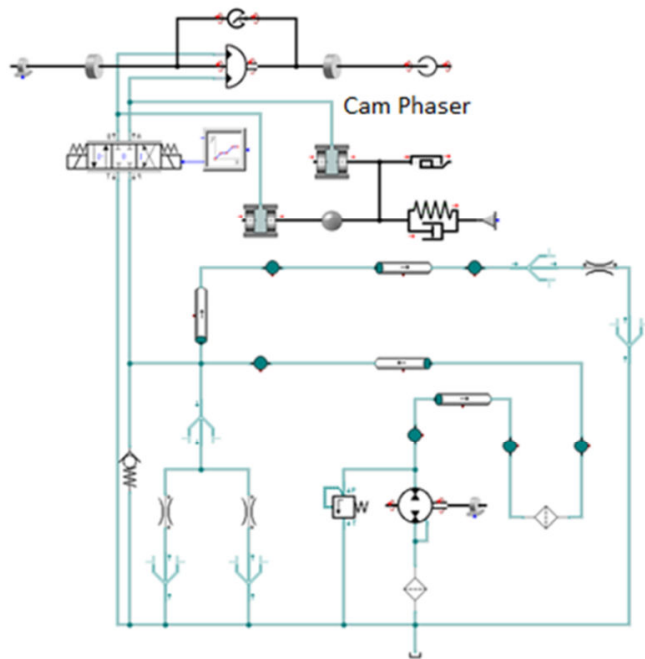

Figure 13 Diagram of Hydraulic Circuit with a Cam Phaser
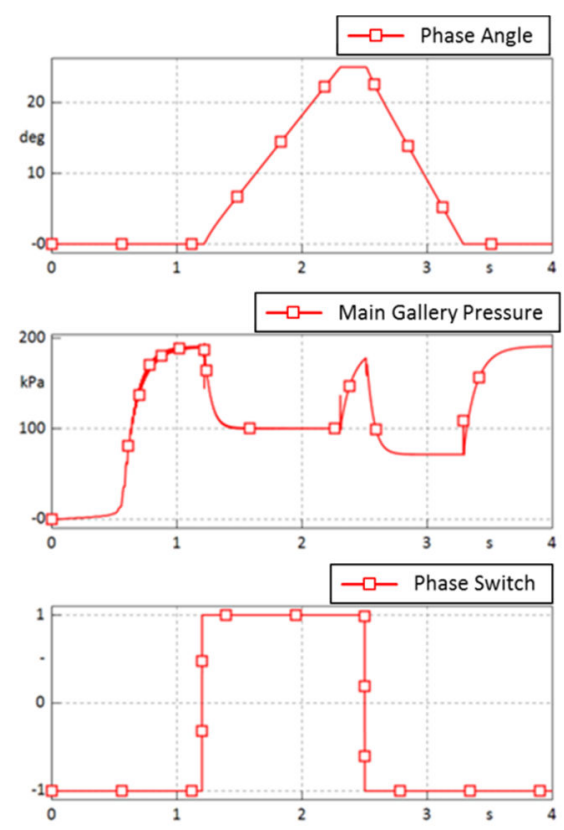

Figure 14 Results of a Single Tool Simulation Model (Top) Phase Angle (Middle) MG Pressure (Bottom) Phase Switch Signal

To branch the circuit at $\mathrm{A}$ in Figure 12, we added a flow source and a pressure sensor in the existing model to send the pressure signal to the sub-circuit model and receive the mass (or volumetric) flow out to the subcircuit (Figure 15). The sub-circuit model has a pressure source as its input and a flow sensor is also added as an output of the model to send the mass flow rate signal to the main circuit model (Figure 16). The total model is shown in Figure 17. 


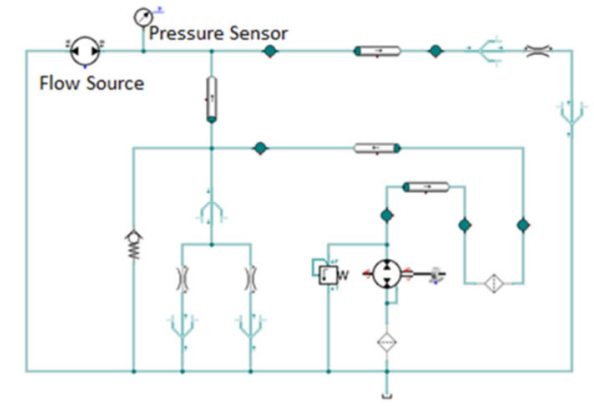

Figure 15 Modified Existing Hydraulic Circuit Model for CoSimulation

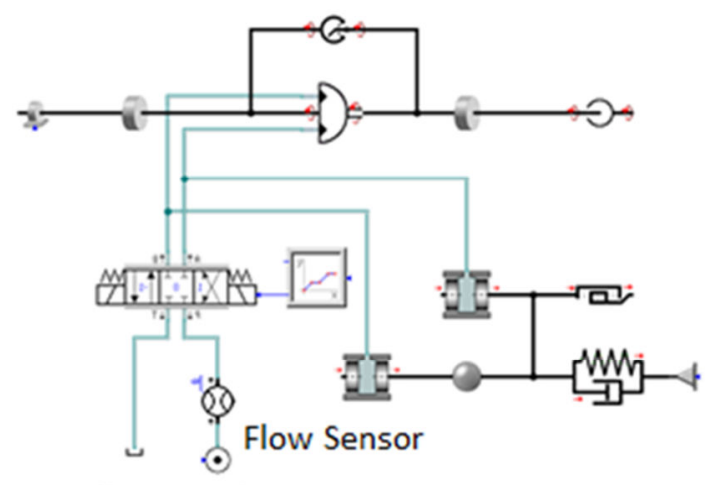

Pressure Source

Figure 16 Connectors of Cam Phaser Circuit

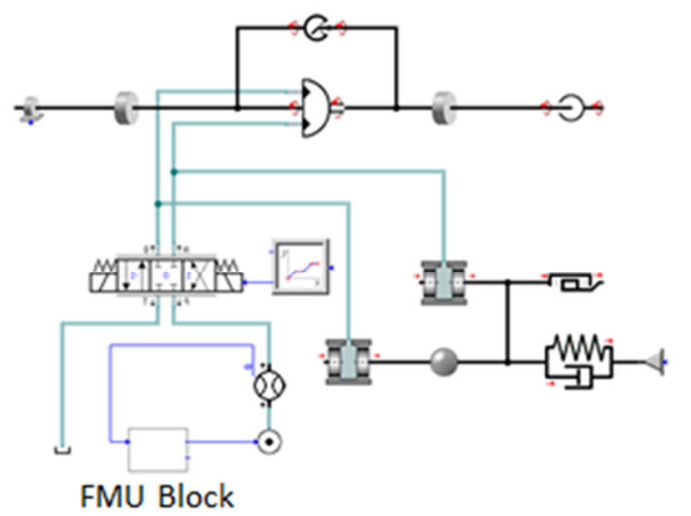

Figure 17 Diagram of Master Tool with a Slave FMU

\subsection{FMI CoSimulation Results}

The two models co-simulate using FMI1.0 tool cosimulation. The tool used for the sub-circuit model is now the master tool since it has a capability to import FMUs of FMI co-simulation. Adding communication ports to the conventional tool and creating a wrapper program to be a slave are relatively easy. The results with the co-simulation model are seen in Figure 18. The communication step sizes (CSS) was set to 1.0E-4 [s].
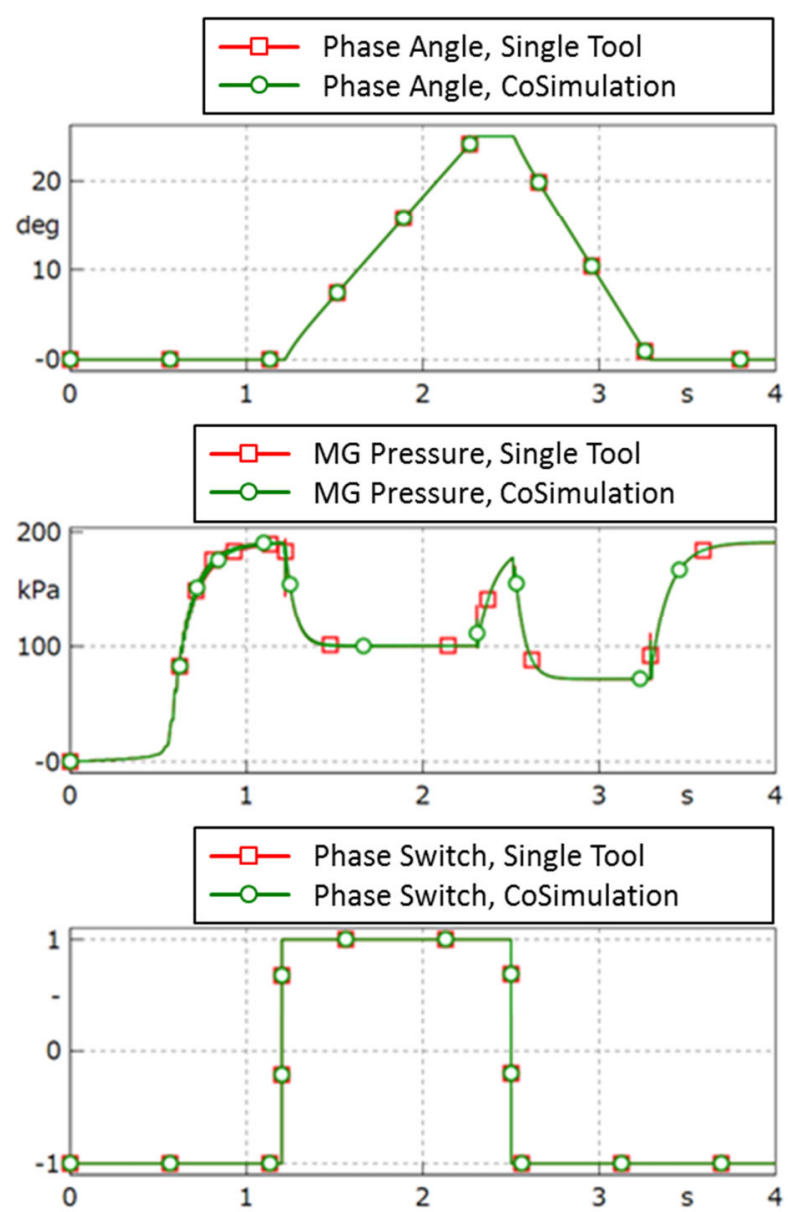

Figure 18 Results of FMI CoSimulation Model and a Single Tool Model (Top) Phase Angle (Middle) MG Pressure (Bottom) Phase Switch Signal

The pressure values at the MG of a single tool simulation and co-simulation are almost identical and so are the phase angles. The differences are less than $0.2 \%$.

\subsection{What We Have Learned}

We confirmed that FMI connection can be used in action and found followings.

1. Fluid property differences have to be discussed beforehand.

2. Initial values should meet but it is not a necessity in this case.

3. Time constant is very small in hydraulic systems. The CSS must be taken into consideration to obtain good results without using much simulation time.

\section{Conclusion}

We employed SimulationX to model mechanical systems used in advanced ICE mechanisms to increase efficiency of ICEs. This kind of simulation can be performed without $\mathrm{CAD}$ data and is useful to confirm 
the availability of new mechanisms at an earlier stage of a developments process.

In the latter half of this paper, a co-simulation of a conventional hydraulic tool and a multi domain Modelica tool is discussed. FMI was used and it was revealed to be applicable for the co-simulation.

\section{References}

Shunichi Aoyama (2009): A Study of A Mulitiple-Link Variable Compression Ratio System for Improving Engine Performance. JSME Journal, Vol.112, No.1092, 2009.

Ryutaro Tagishi et al. (2008) : Development of i-VTEC Gasoline Engine for 2008 Model Year Honda FIT. Honda $R \& D$ Technical Review, Vol.20, No.1 :6-13, 2008. 